This PDF is a selection from an out-of-print volume from the National Bureau of Economic Research

Volume Title: Frontiers in Health Policy Research, Volume 3

Volume Author/Editor: Alan M. Garber, editor

Volume Publisher: MIT Press

Volume ISBN: 0-262-57141-2

Volume URL: http://www.nber.org/books/garb00-1

Publication Date: January 2000

Chapter Title: What Does HMO Market Share Measure? Examining Provider Choice Restrictions

Chapter Author: Laurence Baker

Chapter URL: http://www.nber.org/chapters/c9831

Chapter pages in book: (p. 91 - 112) 


\title{
What Does HMO Market Share Measure? Examining Provider Choice Restrictions
}

\author{
Laurence Baker, Stanford University and NBER
}

\section{Executive Summary}

Many discussions of managed care, as well as policy making and research efforts, treat managed care with a broad brush, forcing a wide variety of organizations into a single framework that facilitates discussion but abstracts from a number of key issues. Although it may be instructive to conduct broad discussions about managed care, a more detailed understanding of the specific activities that managed care plans undertake and their likely effects is required to support comprehensive policy efforts. Frequently, however, efforts to be more detailed are hampered by shortcomings in the measurement of managed care.

This paper lays out a framework of the various activities undertaken by managed care organizations that may be helpful in developing further efforts to understand and measure specific plan activities. The paper also discusses and evaluates some common measures. Detailed data on the exact activities of health insurers and managed care plans are not widely available. One commonly used, but less detailed, measure is the percent of the population enrolled in health maintenance organizations (HMOs). Another places markets on a continuum based on the estimated stage of managed care development in the market. Using data from a new survey, this article compares available measures with more detailed data, suggesting that available data on HMO market share seems to accurately reflect HMO activity, but may not capture the prevalence of some underlying activities of plans like having strong restrictions on enrollee choice of providers, and may also not capture the prevalence of other forms of managed care plans (e.g. Preferred Provider Organizations).

These results suggest the importance of distinguishing between the many activities of managed care plans when considering policies that would influence the development of managed care, and when evaluating results from research studies that primarily rely on measures of $\mathrm{HMO}$ activity. Results from studies of HMOs may not apply to other types of managed care plans. Policies that target HMOs, or other managed care plans broadly, may be less effective than policies that target specific activities of plans. 


\section{Introduction}

As managed care has grown in prominence, numerous research efforts and policy discussions have considered its effects on the health care system and health care delivery. Many of these efforts have treated managed care with a broad brush, however, forcing a wide variety of organizations into a single framework that facilitates discussion but abstracts from several key issues. A central goal of managed care is containing the costs of delivering care, but the wide variety of organizations typically lumped together under the umbrella of managed care pursue this goal using combinations of numerous strategies that vary from market to market and from organization to organization. In most cases, these specific strategies and activities will determine the effects of organizations on matters of interest like costs, patient and physician satisfaction, and health outcomes. Although it may be instructive to conduct broad discussions about managed care on some occasions, a more detailed understanding of the specific activities that plans can undertake and their likely effects is required to support comprehensive research and policy efforts. One goal of this article is to begin laying out a framework of the various activities undertaken by managed care organizations that may be helpful in developing additional efforts to understand and measure specific plan activities.

A second goal is to examine available measures of managed care activity. While it would be useful and interesting to have detailed data on the exact activities of health insurers and managed care plans, such data are not widely available. Rather, researchers and policy makers have frequently been forced to use less detailed measures in the implicit hope that they serve as adequate proxies for the use of various cost containment strategies. One commonly used measure is the percentage of the population enrolled in health maintenance organizations (HMOs). Good data on the number of HMOs operating and HMO enrollment in different types of the country are relatively easy to obtain, and many health care data sets contain indicators for patients who are enrolled in HMOs. Another common measure places markets on a continuum based on the estimated stage of managed care development in the market.

While they have often been the only feasible alternatives, these measures have some important drawbacks. One is that it is not clear how accurate even these broad measures are. Available measures of market 
share are based on estimation algorithms that are difficult to validate and have not been widely compared against other data sources. In addition, HMO market share and managed care staging may or may not adequately capture variations in the use of specific cost containment strategies, and the extent to which they do has not been examined. A better understanding of the quality of available HMO market share and market staging data, and the relationship among HMO market share, market staging, and specific plan activities could help focus the interpretation of studies of the effects of managed care and guide policy-making efforts.

This article reports results from analyses of some recent household survey data from the Center for Studying Health Systems Change that provide additional information on managed care activity at the market level, including both broad measures of HMO market share and more detailed measures of the use of various restrictions on provider choices, one type of cost containment activity in which health plans can engage. The analyses examine three issues. First, I compare existing measures of HMO market share and stage of managed care development with data on HMO market share available from the survey in an effort to examine the validity of the various existing measures of HMO market share. Second, I examine the correspondence between broad measures of managed care activity, namely, HMO market share and market staging measures, and the presence of various limitations that plans place on provider choice. Finally, I examine the impact that using more detailed measures of plan activities, rather than broader measures like HMO market share, could have on the results of studies.

\section{Cost Containment Activities of Health Plans}

The most useful measurements of managed care activity would contain data on the specific activities in which plans engage. Managed care plans can pursue cost containment through a wide variety of activities that can be loosely grouped into five categories. First, health plans may define networks of physicians and health care providers with whom they will work. Defining a panel offers managed care plans the advantage of selecting providers with whom they are interested in working as well as the potential to obtain some contracting advantages through which they can sometimes obtain discounts from physicians who would like to be included in the panel. Some plans define rela- 
tively broad networks of affiliated physicians and providers, while other plans focus their efforts on building a relatively narrow panel and carefully managing it to include only those providers whose patterns of practice are most consistent with the plan's goals.

Second, many plans impose restrictions on patient choices of providers. Plans that have formed networks can impose varying degrees of control on the ability of patients to choose providers in and out of the network. Some plans will not pay for care delivered by physicians not included in the approved panel. Other plans simply provide some financial incentives for patients to see physicians on the plan's panel, but they do pay at least part of the bill for out-of-network care. Many plans also regulate the use of specialists, for example, by forcing patients to sign up with a particular primary care physician or group of physicians and then to obtain a referral from this "gatekeeper" physician or group when specialized services are required.

Third, plans can impose direct controls on utilization. For example, patients and their physicians may be forced to obtain preapproval from a utilization review organization for diagnostic tests, surgical procedures, and other services, if the plan is to pay for the services. Plans can then deny approval for services that they deem to be inappropriate uses of resources. In some cases, direct utilization controls can be imposed in an interactive process with providers. In others, plans simply identify approved and disapproved treatments. For example, mary plans have developed detailed formulas of approved pharmaceutical products for which they will pay, effectively limiting prescribing to the approved list.

Fourth, plans can impose indirect controls on utilization by using financial arrangements that put providers at risk for the financial implications of the patient care decisions they make. For example, many plans use capitation contracts in which physician groups are paid a fixed amount per member per month to care for the patients who have signed up with them. In other cases, plans may withhold a portion of the payments due to physicians and reallocate these funds at the end of the year based on the performance of physicians or groups in meeting utilization, quality, or other targets imposed by the plans. These kinds of incentives may lead physicians to limit utilization without the use of direct central management of utilization.

Finally, many plans engage in efforts to influence physician practice patterns by changing physician opinions about the best ways to care 
for their patients. For example, they may provide information, promulgate guidelines, and define standards for care. Plans may also influence practice patterns by collecting data on the performance of services that the plan (or other observers like the National Center for Quality Assurance) believe to be indicators of quality or of excessive resource use.

Most health plans use more than one of these techniques, and plans vary widely in the combinations of approaches they use and in the weight that they put on each approach. Ideally, measurement efforts would collect data on each type of plan activity. This technique has been difficult but is becoming more feasible over time with emerging databases. Tightness of networks may be identifiable from HMO-level data on the number of physicians in the plans. Restrictions on patient choices are identifiable in some new surveys like the Medical Expenditure Panel Survey (MEPS) and the Community Tracking Study Surveys. Information about the use of capitation appears identifiable from physician surveys.

If the specific activities of plans are not fully measurable, an alternate strategy of pursuing data on a wide variety of health plan model types is also increasingly feasible and should present an improvement over existing HMO market share and market staging measures. To some extent, different combinations of approaches define the stereotypical version of the organizational forms that are commonly observed in the marketplace. Thus, if one can measure the market shares of different kinds of health plans, a significant amount of information about the underlying activities of plans is likely to be gained. Staff and group model HMOs, like Kaiser Permanente, tend to define narrowly a network of providers whose financial incentives are closely aligned with the incentives of the plans and to restrict patients to choosing only providers in the network. Because the panels are carefully defined and financial incentives are already integrated, there is relatively little need to impose strong central controls on utilization or use financial incentives to limit costs. Staff and group HMOs often work hard to define practice guidelines for their physicians.

IPA model HMOs typically have more loosely defined networks of physicians and restrict patients to staying within the network. The looseness of the network and the lack of integration with the plan requires stronger efforts to contain utilization. IPA model HMOs vary in the emphasis they place on using financial incentives as opposed to direct controls, but the prototypical IPA model HMO relies heavily on 
some combination of the two. Preferred provider organizations (PPOs) are characterized by relatively loose panels, some incentives to choose providers in the network (but weaker incentives than those used by HMOs), and limited efforts to control utilization. As a result, PPOs have been regarded as potentially less effective at controlling costs than other organizations. Many formerly unmanaged indemnity plans have also adopted managed care techniques in past years, typically consisting of efforts to impose some (often limited) central control on utilization patterns.

Regardless of how it is measured, obtaining additional information about the prevalence and impact of specific aspects of health plan activities is needed. Different approaches may have different effects on the health care system. Some of these approaches may be more effective at controlling utilization and costs than others. Some may drive physician and patient dissatisfaction more than others. Some may be more or less effective at fostering truly high-quality health care delivery. Identifying strategies that promote the best blend of strategies is an important policy goal. The remainder of this article discusses the measurement of managed care using some new survey data.

\section{Household Survey Data}

In late 1996 and early 1997, the Community Tracking Study Household Survey (hereinafter the CTS) from the Center for Studying Health Systems Change contacted households to inquire about the characteristics of their health insurance. The CTS focused on sixty markets and supplemented the sixty markets with a smaller nationwide sample. In all, data on 60,446 individuals are available. I use data on those individuals residing in one of the sixty focus markets, for a total of 56,798 individuals.

For each household surveyed, data were obtained about insurance coverage for each adult and one of the children if children were present. For each insured individual, the survey asked a series of questions about his or her coverage that can be used to assess exposure to "managed care" and about individual perceptions of the limitations placed on choice of providers. ${ }^{1}$ These questions include:

1. "Does the plan require members to sign up with a certain primary care doctor, group of doctors, or clinic, which they must go to for all of their routine care?" 
2. "Do members need approval or a referral to see a specialist or get special care?"

3. "Is there a book, directory, or list of doctors associated with the plan?" or, for patients with Medicaid, Medicare, or other state insurance, "Can you go to any doctor or clinic who will accept (Medicare/Medicaid/state plan) or must you choose from a book, directory, or list of doctors?

4. "Is the plan an HMO, that is, a health maintenance organization?"

5. For people in plans with lists or in HMOs, the survey asked, "If members do not have a referral, will the plan pay for any of the costs of visits to doctors who are not associated with the plan/part of the HMO?"

Using questions 1, 2, 3, and 5, I computed a variable that indicates the presence of all of the possible restrictions ("very restrictive plan") and a variable that indicates the presence of one or more of the restrictions but not all of the restrictions ("somewhat restrictive plan").

Responses to these questions reflect individuals' perceptions of the characteristics of their plans. Respondents may or may not be fully knowledgeable about the detailed characteristics of their plans. The Community Tracking Study does contain efforts to obtain additional information about the plans from alternate sources, like the written materials provided by each plan. Some preliminary analyses on these materials suggest that responses to the question about whether the plan is an HMO or not tend to be relatively accurate. But responses to the other questions about restrictions are less likely to be accurate. These analyses suggest the importance of viewing the data on plan restrictiveness as reflecting patient perceptions rather than necessarily the actual characteristics of plans.

Some analyses are conducted at the individual level; others are at the market level. The sixty markets targeted by the CTS include fifty-one metropolitan statistical areas (MSAs) and nine other non-MSA market areas. Sample sizes vary across the sites surveyed. Twelve of the sites are "high density" sites and contain relatively large samples, and the remaining forty-eight sites contain smaller samples. The minimum market sample size for analyses using all individuals is 445 individuals. For the market level analysis, I computed market-level measures of the percentage of respondents who reported being enrolled in an HMO 
and the percentage who report having the various restrictions on provider choice for each of the market areas represented in the CTS. ${ }^{2}$

\section{Comparing Existing Managed Care Measures to CTS Data}

\section{Interstudy Data and Market Staging}

Existing studies of the effects of managed care have frequently used measures of HMO market share based on surveys of HMOs, or market staging data, but the accuracy of these data have not been carefully assessed. This section compares these measures to data on HMO market share obtained from the CTS. While the CTS data are themselves estimates, observing a strong correspondence between existing data and CTS data, which are derived from different methodologies, would support the view that both existing measures and CTS measures assess HMO market share accurately.

A commonly used measure of HMO market share comes from the Interstudy Competitive Edge Regional Market Analysis, which reports estimates of the number of individuals enrolled in HMOs in many MSAs (Interstudy 1997). These estimates are derived from HMO reports of the number of their enrollees and the market area that they serve. To obtain estimates for the MSAs, Interstudy apportions the enrollees among the MSAs in the HMO's market area using data on area populations and HMO reports about the distribution of their enrollment across MSAs. These measures are similar to measures that have been used in the past by other researchers (e.g., Baker 1997, 1999, and Wholey et al. 1997).

It was possible to link 1996 HMO market share estimates from the Interstudy Competitive Edge Regional Market Analysis, version 7.1, to CTS HMO market share estimates for fifty markets. ${ }^{3}$ Figure 4.1 plots the relationship between Interstudy and CTS estimates. The Interstudy estimates and estimates from the CTS survey are well correlated ( $r=0.82)$, but the Interstudy estimates are systematically higher. The mean Interstudy market share is 41 percent, while the mean CTS market share is 29 percent, and most of the observations in the figure are above the 45 degree line. A regression of CTS market share on Interstudy market share yields $C=23.11+0.60 * I$, with $p<0.001$ for both coefficients and $R^{2}$ of 0.68 . More people report being in HMOs than are accounted for in HMO market share estimates based on HMO surveys. Given the accuracy of reporting of HMO enrollment apparent from the CTS follow-up data, this result suggests that HMO survey-based data may 


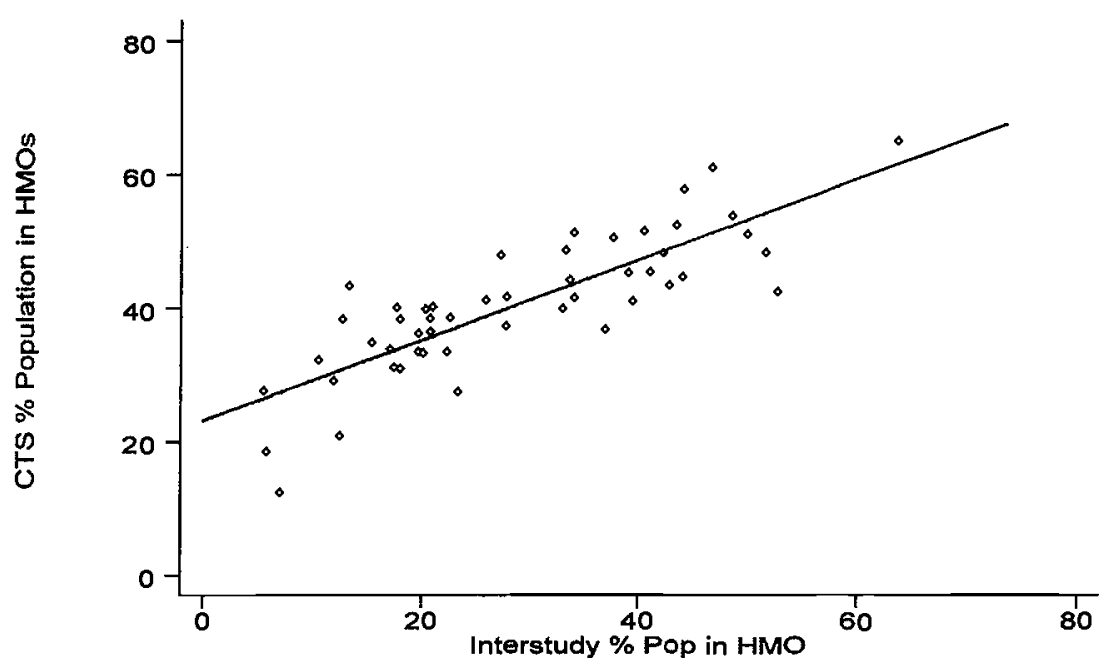

Figure 4.1

Relationship between 1996 Interstudy HMO market share estimates and 1996 market share estimates from the CTS household survey.

Note: $N=50$ MSA markets. Bivariate OLS regression line shown.

understate HMO enrollment in some urban areas. From a research standpoint, the high correlation suggests that both estimates should produce similar qualitative results, perhaps subject to differences in the scale of the coefficients obtained.

Market staging data attempt to assign markets to groups based on the degree of development of managed care in the market. One such measure is developed by the University HealthSystems Consortium (UHC). For various markets, they compile information on a range of market characteristics, including, for example, HMO market share, the number of HMOs with more than 100,000 members, hospital occupancy, hospital days per 1,000 members, number of hospital beds in systems, and the number of state employees in HMOs. The specific pieces of information included vary from year to year. Based on these data, they assign markets a score between 1 and 4 , with 4 representing higher levels of managed care development.

Market staging data have some important drawbacks for research (Burns et al. 1997). The methods used to stage markets are ad hoc and can be hard to discern. Because they incorporate a wide range of information into the staging calculation, there is a risk that the dependent variables in some analyses may be included among the determinants of the staging data. The algorithms and data used to define the stages can 


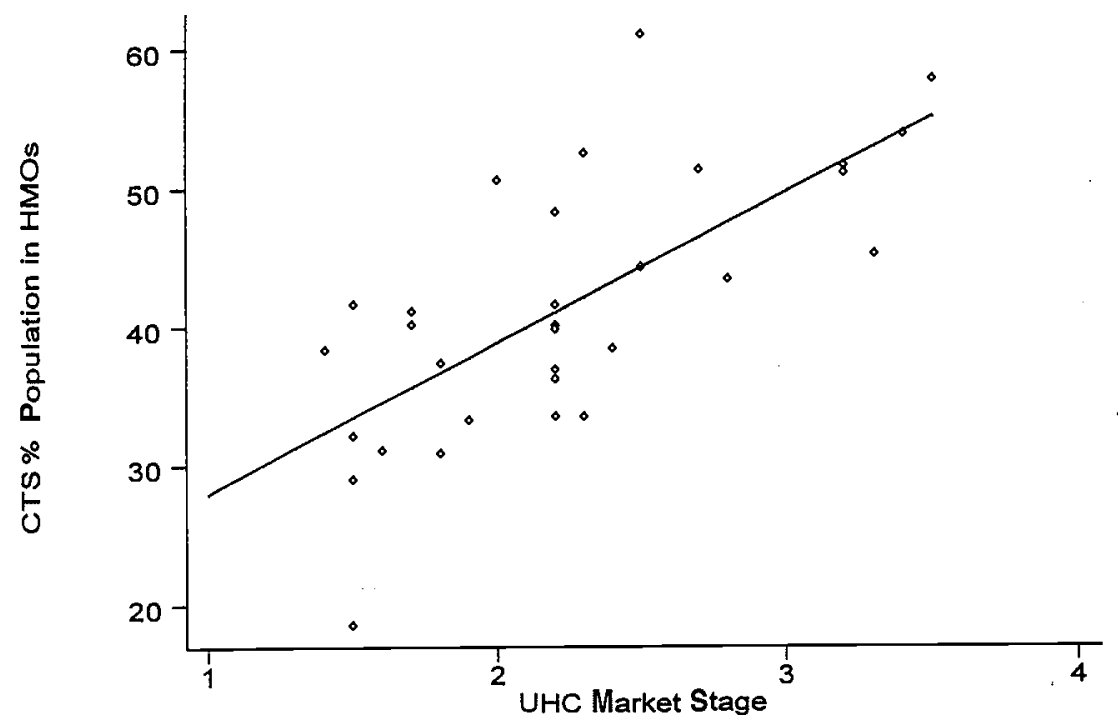

Figure 4.2

Relationship between 1996 UHC market stage estimate and 1996 market share estimates from the CTS household survey

Note: $N=31$ MSA markets. Bivariate OLS regression line shown.

also change over time, making it difficult to do time series work with staging estimates. Nonetheless, some researchers have used staging data in their analyses, and assessing their relationship with HMO market share data and with the presence of restrictions on provider choice seems useful.

Figure 4.2 plots the relationship between CTS HMO market share estimates and UHC's staging data from 1996 for thirty-one markets in which there are both CTS and UHC data. As above, the two measures are positively correlated $(r=0.70)$, and a regression of CTS market share on stage yields $C=17.16+10.85 * U$, with $p<0.01$ for both coefficients and $R^{2}=0.49$. The positive correlation is not surprising because the UHC market staging data incorporate HMO market share estimates. The fact that the $R^{2}$ value is lower here than it is with the Interstudy data indicates a less tight relationship.

\section{Medicare HMO Market Share}

The value of comparisons with the CTS HMO market share data increases with the accuracy of the CTS data. One way to learn more about 


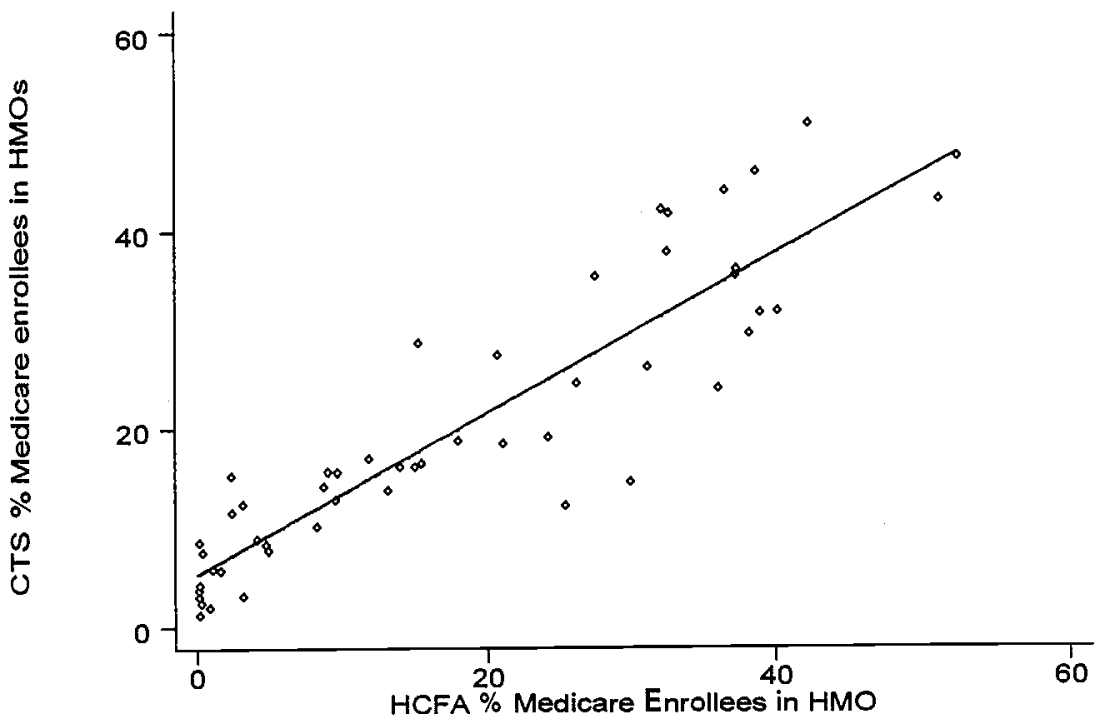

Figure 4.3

Relationship between 1996 HCFA Medicare HMO market share estimates and 1996 Medicare HMO market share estimates from the CTS household survey Note: $N=51$ MSA markets. Bivariate OLS regression line shown.

the accuracy of self-reported HMO membership in the CTS survey is to compare CTS survey data for Medicare enrollees to Health Care Financing Administration (HCFA) data on Medicare HMO enrollment. Because it administers payments for managed care plans, HCFA keeps accurate accounts of the number of Medicare beneficiaries enrolled in HMOs, and thus the HCFA data provide useful benchmarks against which CTS survey data can be compared.

Figure 4.3 plots Medicare HMO market share against CTS HMO market share for Medicare enrollees. The average market shares across the fifty-one markets are about the same: 18.2 percent in the HCFA data, 20.1 percent in the CTS data. Some discrepancy is understandable because there are several markets in which relatively few Medicare beneficiaries were surveyed by the CTS, and hence some measurement error is to be expected. ${ }^{4}$ The correlation between the two measures is 0.91. A regression of HCFA Medicare market share on CTS Medicare market share yields $C=5.32+0.81 \mathrm{H}$, with $p<0.001$ on both coefficients and $R^{2}=0.83$. This result is consistent with the view that the CTS-based survey data reflect actual HMO market shares closely. 
Before extrapolating this finding to non-Medicare populations, however, one should remember that the accuracy of survey responses by Medicare HMO enrollees, who face a clear choice between joining an HMO and staying in FFS Medicare, may be higher than the accuracy of responses among privately insured individuals who are confronted with a set of insurance choices that are often not as well delineated.

\section{Correspondence Between HMO Enrollment and Restrictions on} Provider Choice

Two important cost containment activities that plans can engage in are defining networks of providers and creating policies that restrict patient choices of providers. This section examines the relationship between broad managed care measures and the presence of these types of restrictions, which for ease of discussion I jointly term "provider choice restrictions." First, I examine the relationship between an individual reporting that he or she has joined an HMO and responses to questions about restrictions on provider choice using individual level data. Second, I assess the relationship between area-level measures of HMO market share and the restrictiveness of health plans.

\section{Individual-Level Survey Data}

Table 4.1 reports results from individual-level comparisons between indicating HMO membership and indicating the presence of various restrictions on provider choice. The left-hand side of the table reports results for all respondents. Responses to the HMO enrollment question provide information about the perceived presence of restrictions on provider choice. For each of the individual provider restrictions examined, between 70 and 80 percent of respondents fall on the diagonal of the $2 \times 2$ matrix classifying indicated HMO membership and indicated presence of restrictions. In all four cases, the majority of the off-diagonal respondents are those who said that they were not in an HMO but who did indicate the presence of a restriction. This is understandable given the number of non-HMO managed care organizations that use various restrictions. It also suggests that reporting that one is a member of an HMO is a good indicator that one does perceive the presence of restrictions on provider choice.

The last three rows show the correspondence between indicating HMO membership and indicating membership in a "very restrictive," 
๕ั)

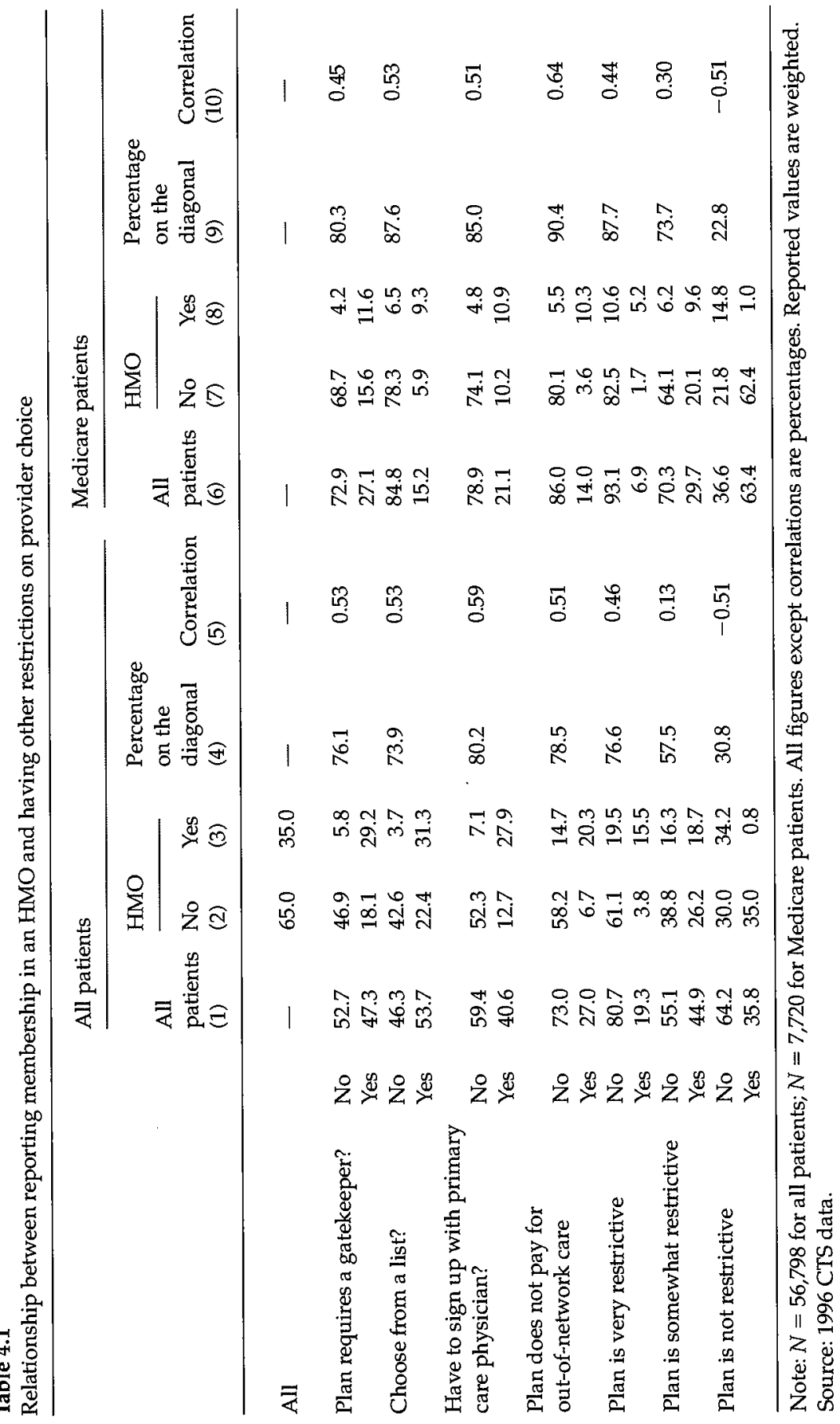


"somewhat restrictive," or "non-restrictive" plan. The relationships here are not as clear. Perhaps the clearest lesson that can be drawn is that an indication of not being a member of an HMO is a strong predictor that an individual does not perceive him- or herself to be in a very restrictive plan. Conversely, indicating HMO enrollment is a strong predictor of perceiving that there are at least some restrictions on provider choice.

The right-hand side of the table repeats the analyses using only Medicare enrollees, who may have a better grasp of whether or not they are actually enrolled in an HMO. Here, results are similar but with even stronger relationships between indicating HMO enrollment and the presence of restrictions. Medicare beneficiaries face many fewer choices of non-HMO organizations than those covered outside Medicare, which may explain some of the difference.

It is interesting to note that a nontrivial number of traditional Medicare beneficiaries (not enrolled in HMOs) indicated that they had a gatekeeper and that they had to sign up with a primary care provider. These reports suggest that there are differences between perceived and real restrictions on provider choice because the traditional Medicare program does not require gatekeepers or signing up with a primary care physician. Overall, these results do suggest that individual-level data on HMO enrollment in studies will provide some useful information about the perceived presence of restrictions on provider choice, although it may not fully capture variation in this dimension.

\section{Market-Area Data}

Many studies examine market-level data instead of individual level data. Aggregated, market-level relationships between HMO enrollment and the presence of provider choice restrictions may differ from the individual-level relationships discussed above. To examine this difference, I compared the market-level measures of HMO market share and provider choice restrictions from the CTS. I also compared CTS provider choice restriction data to Interstudy $\mathrm{HMO}$ measures, UHC market staging data, and HCFA data on HMO market share for Medicare beneficiaries.

Figures 4.4 through 4.6 graph the relationships between CTS HMO market share and the percentage of the population who indicate being in a very restrictive, somewhat restrictive, and nonrestrictive plan, re- 


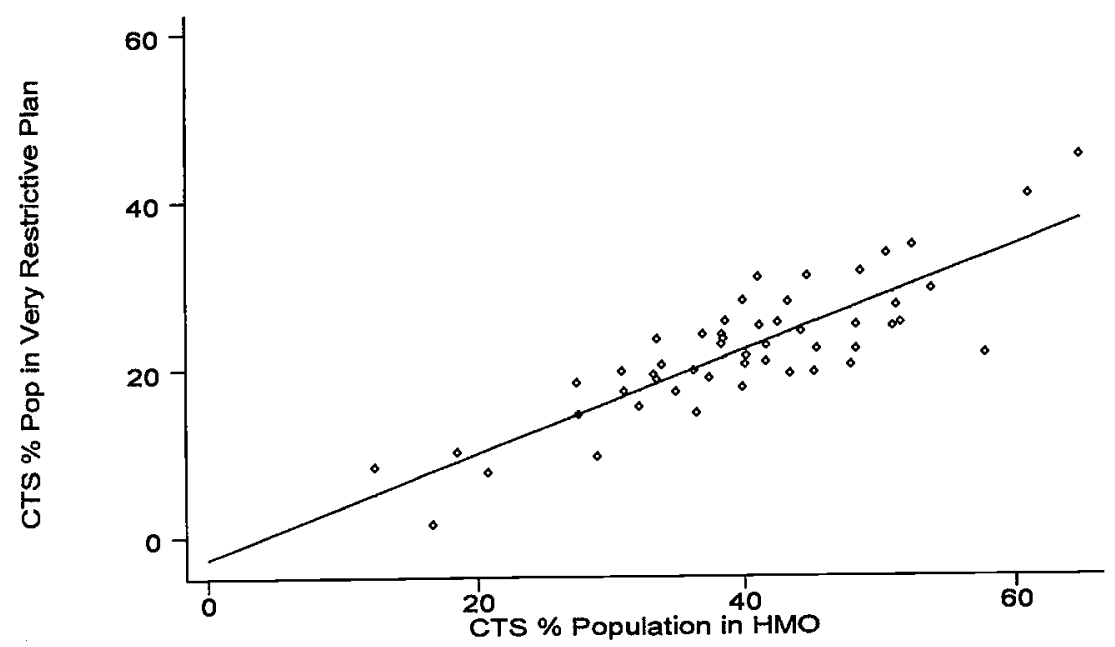

Figure 4.4

Relationship between CTS HMO market share estimates and CTS estimate of percentage of population in a very restrictive plan

Note: $N=60$ CTS markets. Bivariate OLS regression line shown.

spectively. Market-level means of the various provider choice restrictions and their correlations with CTS, Interstudy, UHC, and HCFA measures of managed care activity are shown in table 4.2 for all patients on the left and Medicare patients on the right.

HMO market share estimates from CTS and Interstudy are very positively correlated with the percentage of the population who say that they are enrolled in very restrictive plans, and very negatively correlated with the percentage of the population who say that they are enrolled in nonrestrictive plans. There is not a strong relationship between HMO market share and the percentage of the population in somewhat restrictive plans. The relationship between UHC market staging measures and provider choice restrictions is generally weaker. Staging measures are also correlated with both very restrictive and somewhat restrictive plans. For Medicare patients, where the HMO and provider choice restriction data is probably better, the observed correlations are stronger.

Overall, these results suggest that market level measures of HMO market share are likely to reflect underlying perceptions of the presence of strong restrictions on provider choice but may not capture as well the presence of a smaller set of restrictions. Market staging mea- 


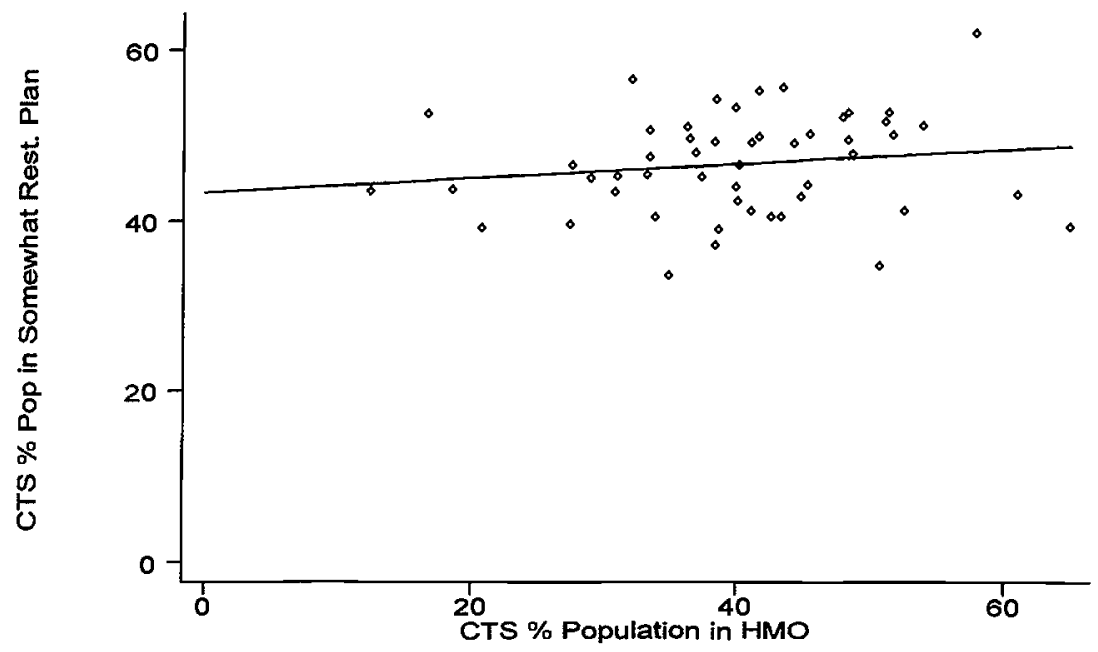

Figure 4.5

Relationship between CTS HMO market share estimates and CTS estimate of percentage of population in a somewhat restrictive plan

Note: $N=60$ CTS markets. Bivariate OLS regression line shown.

sures like those from the UHC may not do as good a job of capturing the presence of provider choice restrictions as $\mathrm{HMO}$ market share measures.

\section{Implications of Using HMO Market Share in Market Studies}

A key question for research and policy concerns the implications of results from research studies that use different measures of managed care activity. Many studies have examined the effects of managed care on health expenditures (e.g., Baker 1999), on technology adoption (e.g., Cutler and Sheiner 1998), and other aspects of the health care delivery system, relying on measures of HMO market share. At a deeper level, however, the hypotheses that drive these studies derive from various specific activities of health plans, like the strength of network arrangements, the use of provider choice restrictions, and the use of capitation, not from HMO market share per se. Thus, a better understanding of the extent to which HMO market share and other measures of managed care activity are related to the underlying activities of health plans can help guide interpretation of results from existing studies. 


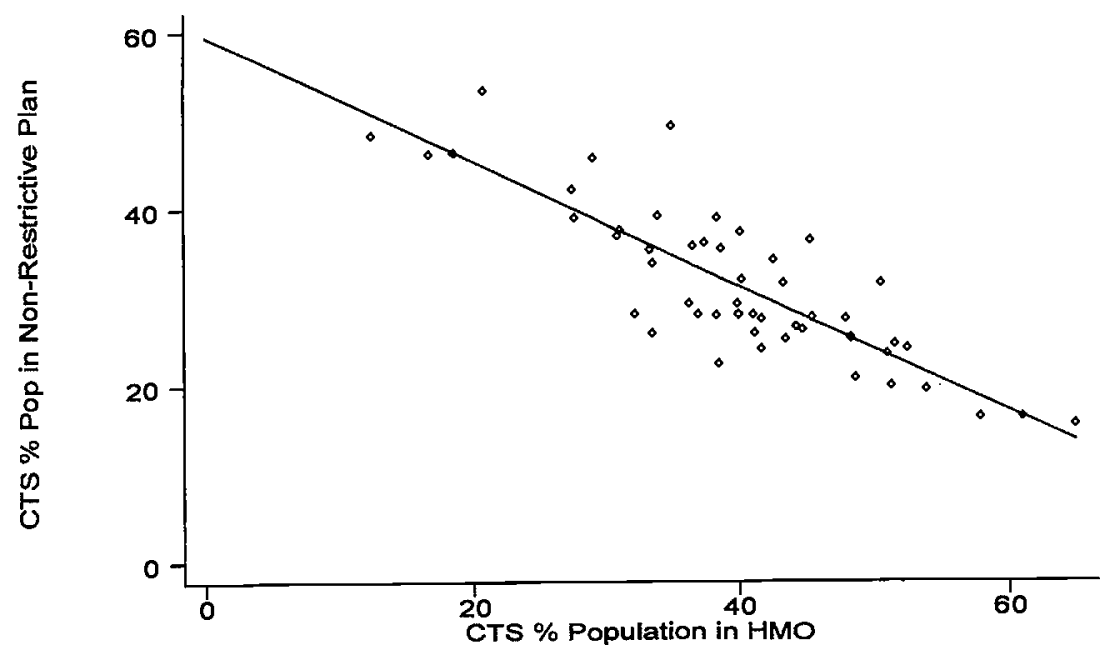

Figure 4.6

Relationship between CTS HMO market share estimates and CTS estimate of percentage of population in a nonrestrictive plan

Note: $N=60$ CTS markets. Bivariate OLS regression line shown.

Results presented above suggest that various measures of $\mathrm{HMO}$ market share should produce similar results, and that HMO market share should do a reasonable job of reflecting the perceived presence of various provider choice restrictions. However, HMO market share was correlated most closely with the perception that plans were "very restrictive" but not with the perception that plans were "somewhat restrictive." Thus, results from studies of HMO market share may be best interpreted as reflecting the effects of having very restrictive rather than somewhat restrictive plans in the area.

To illustrate, this section revisits a previous study of the effects of managed care on the availability of MRI. Baker and Wheeler (1998) summarize some results from a project designed to investigate the relationship between HMO market share and the availability of MRI units in the mid-1990s. Their article used cross-sectional regressions in which the dependent variable measured the number of MRI machines per capita in market areas and the key independent variable was the HMO market share in the area. Other controls for demographics, rural and urban areas, and the like, were also included. The main finding was that increases in HMO activity are statistically significantly associated with reductions in the availability of MRI equipment in 1995. 


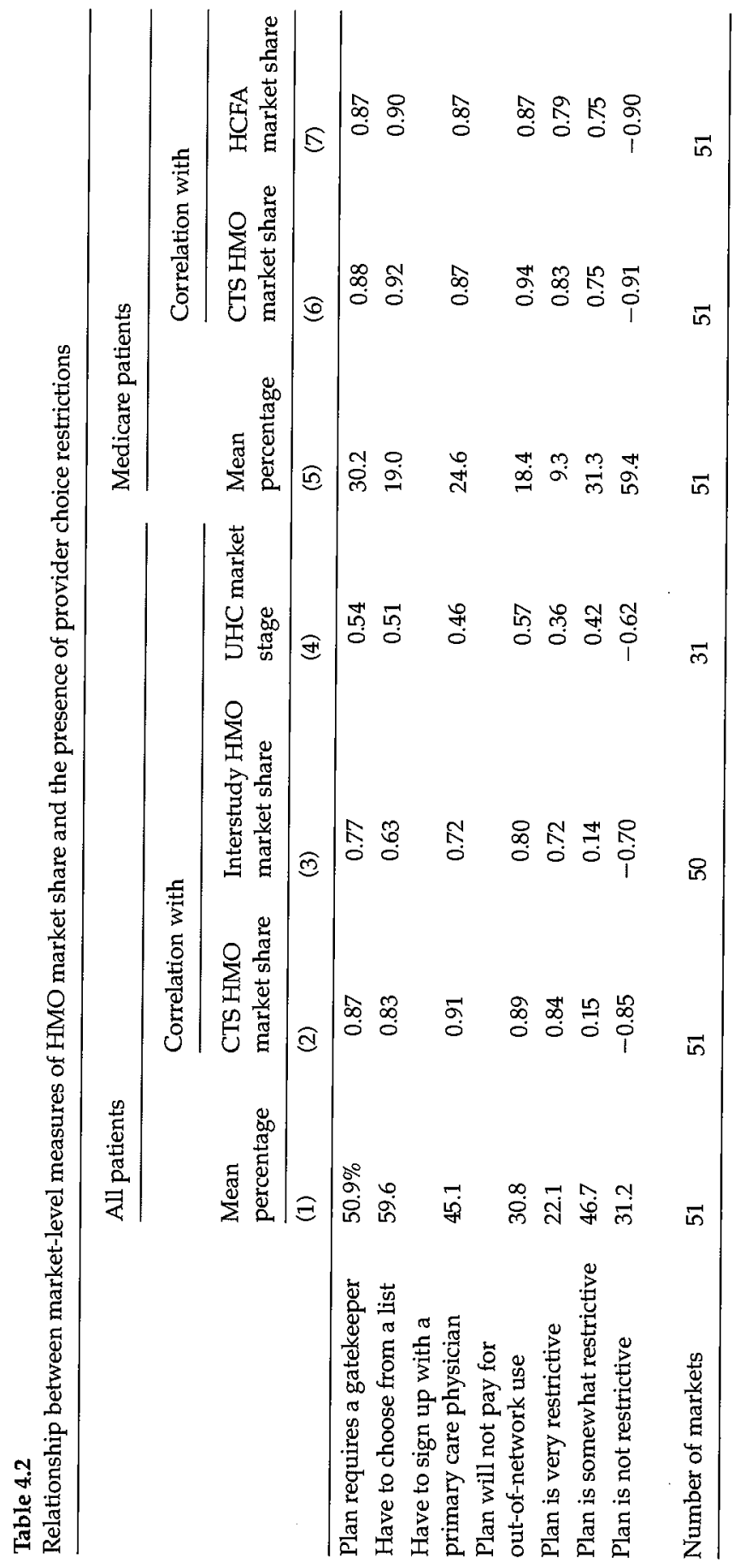


Here, I repeat some of the earlier models with new measures of managed care and the presence of provider choice restrictions. From the original data set, I extracted data on the fifty-one MSA market areas included in the CTS data set, and estimated models in which the various CTS measures of provider choice restrictions (in the entire population) are used to explain the number of MRI sites per 100,000 populations in 1995. None of the estimated coefficients are statistically significant, perhaps because of the very small sample size. Nonetheless, the results do illustrate the variations in results that can be associated with using different measures.

Results are summarized in table 4.3. Using the 1994 HMO market share measure used in the original study, the coefficient point estimates suggest that increases in HMO market share are associated with reductions in MRI availability. Similar results are obtained when the percentage of individuals enrolled in HMOs in 1996 from the CTS is used.

When the percentages of the population in very restrictive and somewhat restrictive plans are used, the results suggest that increases in the percentage in very restrictive plans is associated with declines in MRI availability, but the percentage in somewhat restrictive plans is not associated with changes in MRI availability. The percentage of patients who have a gatekeeper and the percentage of patients who have to sign up with a primary care physician or group have relatively large negative correlations with the number of MRIs, but the percentage of patients who have to choose from a list of providers has a small positive coefficient.

This analysis is not intended to serve as a careful study of MRI availability but to illustrate the implications of better measurement of managed care activity. Without detailed data, one might be tempted to extrapolate from just HMO market share to conclude that the presence of "managed care" reduced MRI availability more generally. But the more detailed data suggest that the HMO effect observed is associated most closely with very restrictive plans with multiple restrictions, with the presence of gatekeepers, and with the requirement that individuals sign up with primary care physicians. Stereotyping plans, this evidence might suggest that HMOs, but not looser organizational forms like PPOs, are associated with reductions in MRI availability. If one were thinking of targeting regulatory or policy efforts at managed care with the intention of influencing technology diffusion, distinctions between organizations and/or the specific managed care activities may be important. More broadly, similar effects may hold for patient and 


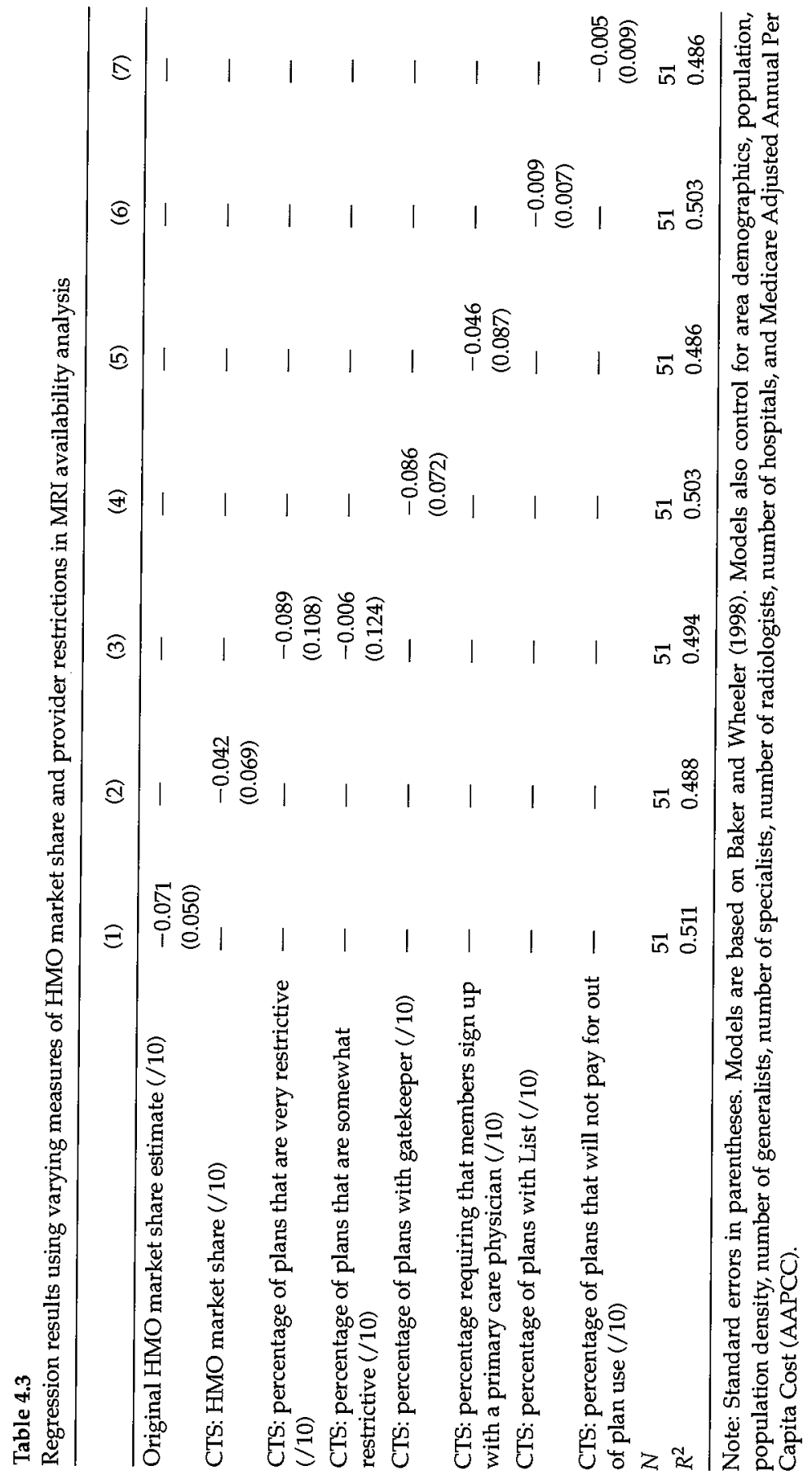


physician satisfaction, health care costs, health outcomes, and other issues of interest.

\section{Conclusions}

Comparisons of data from various sources suggest that some existing measures of HMO market share are well correlated with new survey-based measures of HMO activity, and that HMO market share does convey a significant amount of information about the presence of restrictions on provider choice, particularly the presence of the set of provider choice restrictions that here define very restrictive plans. Research results that use HMO market share are thus likely to reflect the effects of more and more stringent restrictions on provider choice rather than the presence of just a few, less stringent restrictions.

Market staging measures are also used in some studies. Here, these measures were correlated with HMO market share measures and with the presence of provider choice restrictions. The relationship is weaker, however, which leave results from studies using market staging perhaps less easy to interpret as indicators of the presence of provider choice restrictions.

Although many discussions have focused on measures of HMO market share, making finer distinctions between health plans based on the kinds of activities they undertake could help target policy-making activities more accurately. Different health plan activities may have different effects on satisfaction, costs, quality, and other aspects of plan performance, and may be influenced best by specifically targeted policy interventions. The data needed to support studies of the activities of plans has been sparse but are becoming more easily available and should be explored.

\section{Notes}

Prepared for the Third Annual NBER Frontiers in Health Policy Research conference, Washington DC, June 17, 1999.

I am grateful for the excellent assistance of Michael Knisley and Nadia Kapp. This work was supported by a grant from the Robert Wood Johnson Foundation Health Care Financing and Organization ( $\mathrm{HCFO}$ ) program.

1. For persons in families with private insurance, the survey asked about up to three private plans, and I included information from all three plans if present. For example, an individual enrolled in three plans is considered to be enrolled in an HMO if he or she indicated that any of the three plans was an HMO. In the data, 84.5 percent of respondents have one plan, 14.7 percent have two plans, and 0.8 percent have three plans. 
2. The site-level measures are weighted to adjust for sampling patterns and nonresponse within each site.

3. Nine of the sixty CTS markets consisted of groups of counties that did not constitute an MSA and hence could not be linked to the Interstudy data. In one case (Dothan), Interstudy did not report an estimate, so I use fifty observations for this comparison. Since the Interstudy data include everyone in the market, the CTS measures used here reflect HMO enrollment and provider choice restrictions for everyone in the CTS survey.

4. The minimum number of observations per market data set is 45 . The number of observations in the median market is 86 . The correlation between CTS and HCFA data using only the 33 markets with at least 75 observations is 0.94 .

\section{References}

Baker, L. C., 1997. "The Effect of HMOs on Fee-for-Service Health Care Expenditures: Evidence from Medicare." Journal of Health Economics 16(4) (August): 453-481.

Baker, L. C., 1999. "Association of Managed Care Market Share and Health Expenditures for Fee-for-Service Medicare Patients." Joumal of the American Medical Association 281(5) (February 3): 432-437.

Baker, L. C., and S. K. Wheeler, 1998. "Managed Care and Technology Diffusion: The Case of MRI." Health Affairs 17(5) (September/October): 195-207.

Burns, L. R., G. J. Bazzoli, L. Dynan, and D. R. Wholey, 1997. "Managed Care, Market Stages, and Integrated Delivery Systems: Is There a Relationship?" Health Affairs 16(6) (November/December): 204-218.

Cutler, D. M., and L. Sheiner, 1998. "Managed Care and the Growth of Medical Expenditures." Frontiers in Health Policy Research 1: 77-116.

Interstudy, 1997. Competitive Edge, version 7.1. Interstudy: Excelsior, MN.

Wholey, D. R., J. B. Christianson, J. Engberg, and C. Bryce, 1997. "HMO Market Structure and Performance: 1985-1995." Health Affairs 16(6) (November-December): 75-84. 